\title{
Pedagogía, diversidad y lenguaje: develando los colores en miradas aprendientes
}

\author{
Ileana Castillo Cedeño ${ }^{1}$ \\ Centro de Investigación y Docencia en Educación (CIDE), Universidad Nacional \\ Heredia, Costa Rica \\ Luz Emilia Flores Davis ${ }^{2}$ \\ Centro de Investigación y Docencia en Educación (CIDE), Universidad Nacional \\ Heredia, Costa Rica \\ Rafael Esteban Jiménez Corrales ${ }^{3}$ \\ Centro de Investigación y Docencia en Educación (CIDE), Universidad Nacional \\ Heredia, Costa Rica \\ Mariángeles Perearnau Torras ${ }^{4}$ \\ Centro de Investigación y Docencia en Educación (CIDE), Universidad Nacional \\ Heredia, Costa Rica
}

Recibido 26 de octubre de 2009• Aceptado 02 de diciembre de 2009

\begin{abstract}
${ }^{1}$ Doctora en Educación con mención en Mediación Pedagógica. Egresada del Doctorado en Diseño Curricular y Evaluación Educativa, de la Universidad de Valladolid, España. Magíster en Psicopedagogía. Magíster en Educación Preescolar. Especialista en didáctica de la educación del consumidor. Universidad de Valladolid, España. Profesora, bachiller y licenciada en ciencias de la Educación con énfasis en educación preescolar, Universidad Nacional. Actualmente se desempeña como investigadora y ocupa el cargo de Vicedecana del Centro de Investigación y Docencia en Educación de la Universidad Nacional de Costa Rica. Autora de varias publicaciones sobre educación, pedagogía y Universidad en revistas de su especialidad. Correo electrónico: icastill@una.ac.cr

${ }^{2}$ Doctora en Educación con mención en Mediación Pedagógica. Máster en Educación Superior. Catedrática universitaria. Ha ocupado los cargos de Directora de Docencia, Directora de Desarrollo Profesional y Cooperación Universitaria, Presidenta de la Junta de Becas y Coordinadora de la Comisión Técnica de Admisión de la Universidad Nacional. Actualmente, se desempeña como investigadora, extensionista y docente en la División de Educación Básica del Centro de Investigación y Docencia en Educación (CIDE) de la Universidad Nacional, Costa Rica y docente en la Maestría en Formación Dancística del Centro de Investigación, Docencia y Extensión Artística (CIDEA), Universidad Nacional, Costa Rica. Correo electrónico: luzdeflores@yahoo.com

${ }^{3}$ Máster en Evaluación Educativa y Licenciado en Historia. Ha ocupado cargos de Subdirector de la División de Educación Básica del Centro de Investigación y Docencia en Educación (CIDE) de la Universidad Nacional, Costa Rica y Coordinador de la Maestría en Pedagogía con énfasis en Diversidad en los procesos Educativos. Además, formó parte del equipo de Diseño Curricular de la Dirección de Docencia en esa misma institución. Actualmente, se desempeña como docente, investigador en la División de Educación Básica del Centro de Investigación y Docencia en Educación (CIDE) de la Universidad Nacional, Costa Rica. Correo electrónico: rejc21@yahoo.com

${ }^{4}$ Licenciada en Psicología. Ha ocupado distintos cargos en el Instituto sobre Alcoholismo y Farmacodependencia, entre los que destacan: psicóloga de consulta externa, coordinadora de la Clínica Infanto Juvenil y Jefa de Prevención en el nivel nacional. Actualmente, se desempeña como docente e investigadora en la División de Educación Básica del Centro de Investigación y Docencia en Educación (CIDE) de la Universidad Nacional, Costa Rica. Correo electrónico: maperearnau@gmail.com
\end{abstract}

La Revista Electrónic@ Educare está indizada en el Catálogo LATINDEX.

Artículo protegido por la licencia Creative Commons. 
Resumen. En el constante cambio que vivimos, la Pedagogía, en tanto ciencia de la educación, ciencia de la relacionalidad entre seres humanos, es el producto de acuerdos, de procesos comunicativos, procesos socioculturales entrelazados de forma compleja. La atención a la diversidad es una preocupación creciente en ambientes y sociedades plurales, y el lenguaje, como rasgo cultural permite describir nuestras circunstancias y reflexionar sobre ellas para comprenderlas, redimensionar el presente, valorar el pasado e imaginar el futuro. Si tenemos en cuenta que el habla es sólo una de las manifestaciones del lenguaje, si las diversas formas de expresión del cuerpo constituyen formas de lenguaje, se impone la necesidad de pensar cómo nos comunicamos en los procesos pedagógicos.

Palabras clave. Educación, pedagogía, diversidad, lenguaje, comunicación, aprendizaje.

Abstract. In the constant change in which we live in, Pedagogy, as a science of education, science of relationships among human beings, is the product of agreements, of communication processes, sociocultural processes intertwined in a complex manner. The attention to diversity is a growing concern in plural environments and societies, and language, as a cultural trait, allows both the description of our circumstances and the considerations to understand them, to provide new significance to the present, to value the past and to imagine the future. If we take into account that speech is only one of the manifestations of language, if the diverse ways of the body's expression constitute types of language, the need to consider how we communicate in education processes is imposed on us.

Key words. Education, pedagogy, diversity, language, communication, learning.

\section{En la búsqueda de un mundo en el que la palabra permita nombrar a todos los seres vivos}

Vivimos en mundos alternativos, mutables, cambiantes. Esa totalidad que antes llamábamos realidad, no es más que la construcción colectiva de conciencias que llegan a acuerdos sobre miradas y formas de mirar. La pedagogía, en tanto ciencia de la educación, ha sido el producto de esos acuerdos humanos, hijos e hijas de los tiempos, colores distintos producto de la mezcla de la paleta de pintores y pintoras. En ese constante cambio, es imprescindible pensar y reflexionar que los procesos comunicativos se generan entre diferentes interlocutores, éstos miran las relaciones intersubjetivas, las construyen, las crean y recrean según sus historias de vida, sus creencias y sus visiones del mundo.

Nuestras visiones del mundo, nos permiten crear, mirar, reconocer o invisibilizar diferentes entidades vivas. La pedagogía, ya sea en su más elevada concretización teórica, como en la más cotidiana ejecución práctica, es una ciencia, ante todo, de la relacionalidad entre seres vivos; en ese proceso relacional, la comunicación es fundamental, ella es la pintura que muestra la visión del pintor o de la pintora. Según los diferentes artistas, el árbol puede ser verde, rojo, negro, azul; puede ser grande, pequeño o mediano o, bien, el árbol puede existir o no en el paisaje creado. Nos interesa, en especial, que en los procesos pedagógicos todos los seres humanos tengan la posibilidad de ser y de existir, y la existencia humana cobra su vigencia en el tanto que se le nombre, que se le reconozca de forma legítima. Pedagogía, diversidad y comunicación es la forma de reflexionar sobre la necesidad de crear un mundo en el que todos y todas tengan cabida, en el que todos los seres humanos y los seres vivos puedan ser legítimos otros y otras.

\section{Un cíclico y ascendente camino: reconceptualización de la Pedagogía}

El concepto de Pedagogía ha sido construido por autores como Flórez (2005) y Flórez y Tobón (2001), mediante la aplicación de un análisis histórico hermenéutico. Estos autores analizan las 
diferentes manifestaciones de la educación y sistematizan los nodos constantes de análisis sobre el hecho educativo. Conforme ese análisis se va independizando de otras disciplinas va constituyendo un campo específico: la Pedagogía.

Flórez y Tobón (2001) consideran que la Pedagogía es una ciencia en proceso de construcción, no obstante, a pesar de ello, es posible referirse a sus bordes, fronteras, métodos de investigación y campos de acción. Se trata de una ciencia aplicada que se ha ido complejizando conforme se ha complejizado la sociedad en la que le toca actuar.

Básicamente, la Pedagogía estudia los procesos de enseñanza y de aprendizaje. Conforme se han ido analizando y replanteando éstos, la Pedagogía se va modificando, acoplando y resignificando. El punto central de la reflexión pedagógica lo es, sin duda, la formación, la cual se vincula con el tipo de ser humano y de sociedad que se persigue. Por lo anterior, la Pedagogía es un campo de debate con notables matices ideológicos.

El campo disciplinar de la Pedagogía se configura por unas dimensiones o procesos socioculturales denominados, los que, por lo general, se encuentran entrelazados de forma compleja; entre ellos, se pueden visualizar la enseñanza, el aprendizaje, el currículo y la gestión escolar. Para mejorar y accionar dichos procesos, la Pedagogía toma conceptos y teorías que procuran entender y mejorar tales procesos, diferentes métodos de investigación, afines y propios de las ciencias sociales y de otras ciencias, éstos se adaptan al objeto de estudio de la Pedagogía. La Pedagogía es un campo disciplinar con márgenes y contornos abiertos a los aportes de las llamadas "Ciencias de la Educación", las cuales son la miríada de ciencias cuando adaptan su punto de mira hacia la educación. La Pedagogía toma estos aportes, los reconceptualiza, los reconstruye y les da una significación determinada de acuerdo con la respectiva comunidad académica. Flórez y Tobón (2001) resumen de esta forma:

En síntesis, el campo disciplinar de la pedagogía se configura por series de conceptos, teorías y principios generales, modelos y estrategias de enseñanza condiciones de enseñabilidad y diseños de enseñanza de las ciencias en sus aspectos macro y microcurriculares; también por aquellas experiencias de enseñanza en las que se aplica algún concepto o teoría pedagógica, y por supuesto por métodos de construcción de nuevo conocimiento pedagógico teórico o aplicado. (p. 23)

En gran parte de las definiciones sobre Pedagogía, se puede catar un fuerte sabor vinculado con la acción docente, o con las acciones de quien o quienes pretenden enseñar. No obstante, no existiría pedagogía sin la presencia de un ser que aprende: el o la aprendiente. Desde las pedagogías más tradicionales, hasta las más postmodernas, aparece a veces explícito, a veces implícito, el aprendiente o la aprendiente. Toda acción pedagógica cobra sentido, se valida en la acción, en los cambios, en las transformaciones que se generan en el ser del o de la aprendiente. Por ejemplo, en un enfoque netamente tradicional, la acción protagónica del acto pedagógico, en su ejecución, recae en el enseñante, o en la enseñante, pero la verificación de la acción recae en la transformación del aprendiente: Aristóteles fue un gran maestro, pero su aprendiente fue Alejandro Magno. ¿En quién estaba el logro pedagógico? Podría decirse que en la relacionalidad creada entre ambos interlocutores.

Educación y Pedagogía son conceptos que van aparejados, la Pedagogía estudia la educación en sus diferentes manifestaciones, incluyendo el imaginario social que trae aparejada la educación. La Pedagogía nace de una convergencia sintética disciplinaria, la cual es abierta y flexible, Flórez (2005) define la Pedagogía como: 
...término del lenguaje común y más amplio se refiere al saber o discurso sobre la educación como proceso de socialización, de adaptación. En sentido estricto, por pedagogía entendemos el saber riguroso sobre la enseñanza que se ha venido validando sistemáticamente y sistematizando en el siglo xx como una disciplina científica en construcción... (p. 352)

Lo anterior implica que la educación, como fenómeno social, existe antes de la estructuración de la Pedagogía como campo; poco a poco, la educación se va formalizando y sistematizando, con lo cual se inicia una paulatina estructuración del campo pedagógico. Flórez (2005) considera que la Pedagogía inicia su camino autónomo al separarse de la filosofía a principios del siglo xx, con el movimiento de la Escuela Nueva.

La anterior definición implica que la Pedagogía posee su campo intelectual de objeto y metodología de investigación propia, según cada paradigma o modelo pedagógico. Flórez (2005) enfatiza la diferencia de la Pedagogía con la enseñanza y la educación, lo pedagógico incluye la enseñanza y estudia la educación. Tradicionalmente, la Pedagogía ha enfatizado su reflexión en los procesos de enseñanza, pero ello no implica que la reflexión, no haya tenido como eje central el aprendizaje.

La enseñanza, vista desde diferentes posiciones, encuentra su sustento, su razón de ser en el aprendizaje. Desde los modelos de enseñanza más tradicionales hasta los de punta, se validan y logran su consolidación académica en el tanto y en el cuanto, procuren o promuevan aprendizajes, tal y como se ha mencionado anteriormente. Además, en ese sentido, también, se debe repensar en el ámbito de la enseñanza dentro de la Pedagogía, la pregunta básica radica en ¿quién actúa como enseñante en una relación pedagógica? La respuesta dependerá de los supuestos desde dónde se estructure la respuesta.

Nótese que la condición de enseñante más que una concreción en algún ser humano, se trata de una condición situacional y mutable. De esa forma, el o la enseñante puede ser el mismo o la misma aprendiente, la relacionalidad que se establece entre miembros, el medio que rodea al aprendiente y que lo empuja a la constante adaptabilidad o, bien, puede ser cualquier tipo de texto que gatille las conexiones complejas del o de la aprendiente. Creemos que la relacionalidad y la no fragmentación entre enseñante y aprendiente es un adecuado camino por seguir, siempre que el concepto de enseñante se redimensione y supere la mera acción intencionada de un ser humano: "Aprended de los lirios del campo y de los pájaros del cielo", señaló hace unos cuantos años un gran maestro.

El principio general de unificación de la Pedagogía lo constituye el concepto de formación; este concepto debe satisfacer, al menos, tres condiciones, a saber:

- La condición teleológica, que implica el alcanzar siempre niveles superiores para el ser humano.

- La condición metodológica, que incluye procedimientos de investigación, desarrollo conceptual y procesos reales de enseñanza.

- La transformación integral del y de la aprendiente como seres en total relacionalidad (en una dimensión compleja que vincula con la totalidad).

El concepto de formación es el principio clave y unificador de la Pedagogía pero no es el único, ya que una teoría pedagógica debe considerar:

1. Una definición del concepto de persona que se pretende formar, es decir, las metas esenciales de formación humana. 
2. Caracterizar el proceso de formación o el camino de humanización de los jóvenes, en el desarrollo de cada una de las dimensiones constitutivas de la formación en su dinámica y secuencia.

3. Reflexionar, profundamente, sobre el aprendizaje y, sobre quienes aprenden.

4. Describir el tipo de experiencias educativas que se privilegian para afianzar e impulsar el proceso de desarrollo.

5. Describir las regulaciones que permiten enmarcar y cualificar las interpretaciones entre el o la estudiante y el educador o la educadora en la perspectiva de las metas de formación.

6. Describir y prescribir métodos y técnicas utilizables en la práctica educativa como modelos de acción eficaces.

Otras ciencias pueden abordar uno o varios de los elementos anteriores, pero lo hacen de forma eventual. Además, ciertos sentidos sobre cada dimensión se han sedimentado como principios imprescindibles de la moderna Pedagogía.

La forma en cómo se ha ido configurando la Pedagogía, como campo o disciplina, nace de la observación histórica del hecho educativo, es decir, la Pedagogía es una ciencia que nace de la propia acción de educar a lo largo de la historia de la vida.

La Pedagogía es una ciencia en la cual la relación teoría-práctica se presenta como un binomio indisoluble, en el que la formación del ser humano opera como eje estructurante. Una teoría pedagógica consistente generará seres humanos más inteligentes, más autónomos y más universales.

\section{Mundos inacabados: la Pedagogía como campo en construcción}

La Pedagogía se considera un campo en construcción constante por las siguientes razones:

- Posee una susceptibilidad e inestabilidad histórica en su relación y complejidad teóricopráctica.

- Posee un carácter cambiante y multivariado producto de los procesos educativos que estudia (época, sociedad, región, régimen político, ciclo, nivel de enseñanza, entre otros).

\section{Estrellas, atmósferas tierras y mares: niveles de concreción de la Pedagogía} niveles:

La Pedagogía es, además, un campo complejo, ya que se desarrolla o estructura en tres

- I Nivel. Eje de la formación humana. Actúa como principio unificador y sistematizador en el que se ubican las teorías pedagógicas generales, las corrientes, las escuelas de pensamiento y las grandes vertientes conceptuales.

- II Nivel. Intermedio. Está compuesto por la articulación de modelos y conceptos de un nivel medio de abstracción, en él se ubican elementos tales como las representaciones de teorías pedagógicas particulares, las estrategias de enseñanza, las concepciones del aprendizaje, las valoraciones del papel que juegan los y las aprendientes, los diseños macrocurriculares, las condiciones de enseñabilidad de cada ciencia de las que se derivan las llamadas didácticas específicas. 
- III Nivel. De aplicación de conceptos. Este nivel implica la apropiación y la verificación conceptual en la acción pedagógica, se vincula con la acción de la enseñanza y/o del y de la aprendiente. La dinámica de este nivel se genera en la tensión dada entre estudiante, entorno, mundo de la vida y el micro-currículo (específico para determinados estudiantes en sus respectivos entornos vitales).

La Pedagogía, como campo conceptual, implica la articulación interdisciplinaria de estos tres niveles señalados.

\section{Desde el Big Bang, el universo no ha parado de vibrar: las recurrencias formativas en la his- toria como principios pedagógicos}

Desde una posición hermenéutica, la Pedagogía como campo ha sostenido ciertos principios pedagógicos recurrentes en la historia, los cuales han variado de matiz o nivel de intensidad, no obstante, se siguen presentando en los discursos y acciones:

$\sqrt{ }$ El afecto.

$\sqrt{ }$ La actividad.

$\sqrt{ }$ El diseño del ambiente de aprendizaje.

$\sqrt{ }$ El desarrollo progresivo de las capacidades del o de la aprendiente.

$\sqrt{ }$ La experiencia natural como potenciadora de aprendizajes.

$\sqrt{ }$ La individualización.

$\sqrt{ }$ El anti-autoritarismo y el cogobierno (la Pedagogía transita de sistemas totalmente unidireccionales a sistemas coparticipativos).

$\sqrt{ }$ La actividad grupal, equipos de trabajo y comunidades aprendientes.

$\sqrt{ }$ Las actividades lúdicas.

$\sqrt{ }$ La búsqueda de perfilar un ideal de buen o buena docente.

Los anteriores principios no pueden considerarse absolutos ni ahistóricos, están expuestos a la crítica, la refutación; pero ellos se han ido configurando por medio de la historia de la Pedagogía. Ellos constituyen el núcleo teórico de las pedagogías contemporáneas (Flórez, 2005).

Como se señaló anteriormente, el ideal humano y social es pilar básico en la concretización teórica de toda construcción pedagógica; de esa forma, si se visualiza como ideal una sociedad homogenizante, se intentará estructurar el camino pedagógico; por el contrario, si se posee la utopía posible de una sociedad tendiente a la construcción de espacios para la diversidad, así serán las posibles rutas pedagógicas. Ahora bien, esa construcción de una propuesta pedagógica, aun con aspiraciones sociales hacia la diversidad, se vinculará, directamente, con la misma construcción del ideal semántico de la diversidad, recuérdese que el lenguaje es creador de realidades y utopías.

\section{Los borrosos tonos del arco-iris: diversidad}

La atención a la diversidad es una preocupación creciente en ambientes y sociedades plurales en las que se refleja, pese a discursos que abogan por la igualdad, un silenciamiento y negación de diferentes culturas y formas de vida. 
Aunque se reconoce que la diversidad es parte inherente de los procesos de coevolución universal y, por ende, humana, existen múltiples formas de actuar y de pensar que aniquilan la riqueza de la diferencia, sobre todo, cuando se habla de culturas y sus cosmovisiones. Muchas veces, dentro de los procesos formativos, no se tiene la visión de que en el aula coexisten seres humanos cuyas historias multicolores, impiden homogenizar el currículum. Algunos autores reconocen que el aula es un nicho ecológico en el que convive una serie de micro-culturas dignas de considerar.

Desde esta perspectiva, innegablemente, más que disertaciones perentorias relacionadas con la diversidad, se requiere que la sociedad asuma, en forma conjunta con instancias educativas gubernamentales y no gubernamentales, de carácter formal y no formal, compromisos en el plano de la reflexión rigurosa y la acción crítica, que contemplen elementos de índole epistemológico, pedagógico, político, cultural e histórico para que se manifiesten verdaderas transformaciones.

Determinantemente, se requiere de un método permanente de reflexión, análisis y debate que profundice en el conocimiento de los dispositivos de exclusión, marginación, segregación, desde todos los sectores que forman el aparato estatal, de manera especial, centros educativos de formación inicial, primaria, secundaria y universitaria, en los que se evidencian procedimientos y prácticas que lejos de potenciar la pluralidad democrática y la libertad, provocan la exclusión y el autoritarismo.

Los centros educativos de educación superior, por su función social formadora y transformadora, tienen el deber de convertirse en gestores de experiencias de aprendizaje auténticas, en los que la convivencia se convierta en el mayor conocimiento y herramienta para asumir la vida en toda su complejidad y diversidad.

Assman, precisamente, concibe las escuelas, como comunidades de aprendizaje, pero aprendizaje en términos de convivencia. La formación de la persona para su inserción en el ámbito económico y laboral es importante desde el punto de vista profesional y social. No obstante, en este momento, interesa, ante todo, el desarrollo personal, en el que los afectos y las emociones son relevantes. Sólo en esos contextos seremos capaces de apreciar la complejidad y la sencillez de la totalidad, conformada por unidades interdependientes que le dan sentido a la vida.

Evidentemente, al hablar de diversidad como antes se indicó, no basta con establecer vínculos entre minorías étnicas, raciales, culturales; hay elementos de gran complejidad que hay que analizar, por ejemplo, cuando se habla de situaciones como fracaso escolar. Si bien es cierto, estos aspectos deben formar parte de la comprensión, no se debe obviar que la educación es un proceso político y social que privilegia a ciertos grupos de la población.

La diversidad tampoco debe ser asociada a elementos relacionados con la superación de dificultades o problemas de aprendizaje, aspecto que se ha generalizado en los últimos tiempos por la psicología o psicopedagogía, asociados, a su vez, con rendimiento escolar. Ciertamente, la atención a la diversidad debería permitir atender, de manera oportuna, las necesidades educativas especiales mediante la contemplación de diferentes ecologías cognitivas, las que provocan diferentes ritmos y estilos de aprendizaje, de los que emergen formas de resignificación del mundo y la vida disímiles.

\section{Diversidad: apropiación y recreación de la realidad desde la universidad}

La educación, como proyecto social, requiere valorar el funcionamiento de sus instituciones y revisar, cuidadosamente, no sólo los planes y los programas de estudio que fundamentan su quehacer, sino, también, las prácticas pedagógicas que se ejecutan dentro y fuera de las aulas, para adquirir una visión global y real de los alcances y las limitaciones en relación con el vínculo entre teoría y práctica. 
La línea diferenciadora de cualquier institución educativa va a ser la autenticidad con que asuma los desafíos de sociedades altamente complejas, la apertura al cambio, a la crítica y al diálogo. Cada centro educativo, desde su singularidad, debe ir aportando a la construcción de alternativas educativas enraizadas en la diferencia, y ver la diferencia como un elemento dinamizador social, no como una amenaza, sino como una oportunidad para aprender de ella.

En este sentido, mucho tienen que aprender las instancias de formación en el nivel superior, sobre todo, si se reconoce que deben convertirse en comunidades críticas, en las cuales hay que empezar observando hacia dentro para, luego, mirar hacia fuera.

La formación, en este sentido, tendrá que entenderse de forma amplia, no basta sólo con los procesos de formación inicial de docentes en centros o facultades de educación, se trata, también, de la educación continua de docentes en servicio, aspecto que sobrepasa la oferta de cursos aislados. Tendría que ser un proyecto global que involucre a todos los actores y las actoras del proceso educativo.

La perspectiva señalada, implica una visión de aprendizaje permanente y contextual. Como antes se dijo, no es suficiente un excelente diseño curricular; se requiere de decisión y disposición y, sobre todo, de identidad para no dejarse abrumar por las prescripciones políticas que, muchas veces, lejos de potenciar la diversidad, la limitan.

Necesariamente, las universidades deben comprender cuáles son las claves y los significados de cada acción en contextos globales como los que vivimos, sólo de esa manera se podrán perfeccionar y transformar las prácticas. Esto, también, impone claridad con respecto al potencial de cada persona, la valoración de elementos claves, tales como el presupuesto, de manera que se asegure un proceso de calidad y de equidad para las personas.

Desde hace muchos años, se vienen desarrollando experiencias exitosas en el contexto europeo y latinoamericano, de atención a la diversidad, éstas parten de la comprensión de que el currículum no puede ser una imposición social, sino una construcción humana realmente participativa, lo cual da origen a proyectos tanto educativos como curriculares de centro. Esto forma una línea clave de respeto y de atención a la diversidad.

Las organizaciones institucionales tendrían que permitir que aflore la diversidad, admitiendo que ella existe, y que tanto estudiantes como académicos y académicas, así como funcionarias y funcionarios administrativos, deben formar parte de ese proyecto educativo que dé vida a la vida.

Sólo con la reflexión rigurosa y comprometida, podremos comprender los misterios y los significados ocultos dentro de la actividad educativa. La mejora de la atención a la diversidad se dará en forma efectiva y no ficticia o asistencialista como hasta ahora se ha hecho, cuando se permita el aprovechamiento de espacios académicos en un sentido genuino, menos cargados de burocracia y más llenos de organicidad y comunicación, por tanto, de decisiones y acciones compartidas y sentidas, que lleven a la edificación de sueños y esperanzas.

\section{La edificación de los sueños: lenguaje}

El lenguaje constituye una característica fundamental para la comunicación, para compartir y sentir las decisiones y las acciones, para establecer interacciones que posibiliten la vivencia en comunidad.

La teoría del conocimiento de Humberto Maturana, establece que los seres vivos somos sistemas determinados en nuestra estructura, y formula que "el lenguaje no se da en el cuerpo como un conjunto de reglas, sino en el fluir en coordinaciones conductuales consensuales... El lenguaje tiene que ver con coordinaciones de acción, pero no con cualquier coordinación de acción, sino con 
coordinaciones de acciones consensuales". (Maturana, 1989, p. 28). El énfasis, entonces, está en el espacio de relaciones, de acción; por ello, este autor acuña el término "lenguajear" para enfatizar el entrelazamiento entre lenguaje y emociones. Las emociones, en esa teoría, son disposiciones corporales dinámicas que definen los diferentes dominios de acción, por tanto, no se confunden con sentimientos. El lenguaje y las emociones se interrelacionan y al interactuar se modifican mutuamente. Se entiende, entonces, que la vida humana se conforma en una red de conversaciones.

En el marco de esta teoría, el lenguaje se concibe como el origen de lo humano. Se reseña la aparición del lenguaje como herramienta humana para facilitar el compartir, y se postula que las personas existimos en un espacio de relaciones e interactuamos, fundamentalmente, por medio del lenguaje. Zihlman y Tanner (citados por Eisler, 1998), también "atribuyen los primeros vínculos sociales humanos y el origen del lenguaje a la necesidad de madres e hijos de comunicarse" (p.13).

Vivimos como seres humanos en contextos sociales gracias al lenguaje, por tanto, somos seres sociales en el lenguaje. No obstante, para el desarrollo del lenguaje es indispensable que se establezca la consensualidad o las coordinaciones de conductas. El lenguaje surge en la convivencia y posibilita la comprensión recíproca, aspecto medular en un proceso pedagógico.

El lenguaje, como rasgo cultural que pertenece al dominio de la conducta y como fenómeno biológico, nos permite describir nuestras circunstancias y reflexionar sobre ellas para comprenderlas y explicarlas, redimensionar el presente, valorar el pasado e imaginar el futuro; nos posibilita, también, el acceso a la dimensión espiritual.

Como revela Heisenberg (citado por Frei Betto, 1998, p. 207): "la ciencia y el arte son formas de un lenguaje humano que nos permite hablar de los detalles más disimulados de la realidad”. Sin embargo, el mismo autor reconoce, también, que "la física cuántica y la mística tienen en común el hecho de resaltar la insuficiencia del lenguaje”. Surge, entonces, la pregunta: ¿Existe lenguaje en el silencio?

Como se mencionó anteriormente, para Maturana el lenguaje es un fluir de coordinaciones conductuales consensuales. En sus palabras “...el lenguaje es un flujo en coordinaciones recurrentes de conductas, se produce a través de la real operación estructural de nuestras interacciones e involucra una dinámica de cambios estructurales en los participantes que sigue un curso en cada uno de ellos, contingente momento tras momento en el flujo de coordinaciones recurrentes de conducta en las cuales participa”. (Maturana, 1999, p.44). Así pues, el habla es solamente una de las manifestaciones del lenguaje. Todas las formas de expresión que realizamos utilizando nuestro cuerpo constituyen formas de lenguaje: la danza es un bello ejemplo de ello.

Maturana (1999), clarifica que el lenguaje no es un dominio de operación con signos o con símbolos, ni se lleva a cabo mediante ellos, sino, más bien, éstos son secundarios al lenguaje. Para operar con símbolos, nos dice, debemos estar ya en el lenguaje. Según este destacado biólogo, "el lenguaje como núcleo del modo de vida conservado en nuestros antepasados debe haber comenzado hace más de unos tres millones de años" (p.51).

Al entender la importancia del lenguaje en la conformación de nuestro ser humano se acentúa la relevancia del respeto que merece el lenguaje de los y las estudiantes, pues ello es respeto a su existencia, a su hogar, a su medio, a su cultura. La diversidad que caracteriza a las personas participantes en un proceso pedagógico amerita la reflexión respecto a las formas de comunicación que se establecen, tanto mediante el uso del habla como de otras manifestaciones, y, fundamentalmente, la importancia de la comprensión de las intencionalidades que subyacen a las expresiones.

Las diferencias compartidas enriquecen la comunicación. De ahí, el valor de crear espacios para la libre expresión de las conceptuaciones utilizadas en el proceso pedagógico; aspecto, por demás 
indispensable, para conocer las ideas previas del estudiantado, con referencia en las temáticas en estudio.

Las interacciones recurrentes en el espacio del aula entre docente y estudiantes, así como entre estudiantes, generan una historia de formas de relación que configuran las estructuras de las personas participantes. La dinámica que se vive genera cambios de manera congruente con el tipo de relaciones establecidas, y surge un nuevo modo de convivir en el lenguaje.

En este sentido, es primordial tener presente que el lenguaje permite construir significados. Como bien lo han establecido tanto Paulo Freire como Lev Vygotsky, lenguaje y pensamiento se producen simultáneamente. "Todo ello nos lleva a dimensionar la importancia que tiene utilizar un lenguaje inclusivo, no discriminatorio, no sexista, en la construcción de una sociedad gilánica, y la necesidad de invención de términos para nombrar nuevas formas de relación cooperativa." (Flores, 2007, p. 25) Cuando se habla de una sociedad gilánica se hace referencia a una sociedad solidaria, cuya norma de convivencia es la igualdad entre los sexos y, en general, entre los pueblos. Este tipo de sociedad se construye en conspiración desde el lenguaje, desde el establecimiento de relaciones de respeto y de amor con la naturaleza, en general, y con la humanidad, en particular. Y en esta tarea, tiene la Pedagogía uno de sus principales desafíos.

\section{La totalidad en el decir: comunicación no verbal}

Si el habla es sólo una de las manifestaciones del lenguaje, si las diversas formas de expresión del cuerpo constituyen formas de lenguaje, se impone la necesidad de pensar cómo nos comunicamos con nuestros semejantes por medio de sutiles cambios en nuestras expresiones.

Muchas veces, el límite entre la comunicación verbal y la no verbal es muy sutil y poco definido, pero no por ello menos presente. Todos hemos vivenciado situaciones en las que hemos percibido contradicciones entre lo que una persona nos manifestaba, oralmente, y lo que captábamos, realmente. Cuando se plantea este dilema, por lo general, creemos lo que sentimos de forma no verbal.

Mediante diversas investigaciones se ha puesto de manifiesto que nos comunicamos mucho más por medio de comunicación no verbal que lo que hacemos oralmente. Según Mehrabian, en sus investigaciones se manifiesta que sólo el 7\% de la información captada se debe a la comunicación oral, el 38\% se debe a las variaciones de la voz (tono, volumen, entonación,...) y el 55\% restante al lenguaje corporal. En este lenguaje corporal, tendríamos que tener en cuenta las variaciones posturales, los gestos, la expresión facial, le respiración, el sonrojo...

En otros estudios se concluye que, aproximadamente, un treinta por ciento de lo que percibimos lo interiorizamos por medio de la comunicación verbal y cerca de un setenta por ciento se capta por la comunicación no verbal.

Vemos que, en todos los casos, la comunicación no verbal constituye el mayor porcentaje de nuestra comunicación.

Comunicamos en todas las circunstancias. Siempre nuestro comportamiento y expresión transmitirán más de lo que pensamos emitir, ya que, muchas veces, lo que nuestra actitud presenta no es conocido por nosotros mismos o nosotras mismas.

Cuando lo que emitimos es congruente no nos damos cuenta de que percibimos mediante distintos canales, sólo se evidencia cuando sentimos discrepancias en la comunicación. Y cuando se da esta situación, casi siempre, creemos en lo que incorporamos de la comunicación no verbal.

Este lenguaje corporal lo transmitimos inconscientemente, por lo que, en gran medida, nos pasa desapercibido. Sin embargo, todos y todas hemos experimentado una sensación de agrado 
o de desagrado al conocer a una persona, cuando, sin ningún dato, sentimos que una persona es agradable, que nos gustaría entrar en contacto con ella, o percibimos una amenaza y pensamos que no queremos estar en contacto con esta persona, que no queremos trabajar con ella o, simplemente, cancelamos la posibilidad de iniciar una comunicación.

La sociedad de consumo intenta comerciar con todo y, por supuesto, la comunicación no verbal está en su punto de mira. Vemos que proliferan cursos para instruir a las personas sobre su presentación a las entrevistas de selección de personal, se les adoctrina sobre cómo ir vestidos, sobre su forma de dirigirse al entrevistador, sobre la forma de hablar o callar sobre ciertos temas, y no negaremos que estos mini cursos, puedan proporcionar informaciones importantes, para algunas personas, pero hay una dimensión que se escapa a esta comunicación planeada, que es algo más íntimo, está relacionado con las percepciones, las emociones, las sensaciones; las comunicaciones más ocultas.

Tanto en la comunicación verbal como en la no verbal pueden existir distorsiones por parte de cualesquiera de las personas participantes en el contacto. Se pueden lanzar mensajes distorsionados por temor, inseguridad, miedo y las otras personas pueden recibirlos, también, deformados por las mismas emociones que impiden recibir, tranquilamente, lo que la otra persona quiere compartir.

Muchos conflictos personales pueden alterar lo que se escucha o siente, y las necesidades afectivas, pueden, también, distorsionar lo que, en realidad, acontece en una conversación. Se impone, entonces, la necesidad de pensar cómo nos comunicamos en los procesos pedagógicos, pues el mensaje no verbal es tan poderoso que una persona, con una simple mirada, puede hacer sentir mal o puede estimular a emprender grandes desafíos.

\section{Referencias bibliográficas:}

Eisler, R. (1998). Placer sagrado. Nuevos caminos hacia el empoderamiento y el amor. Volumen 2. Santiago, Chile: Editorial Cuatro Vientos.

Flores, L. E. (2007). Educación y equidad de género. San José, Costa Rica: Universidad de La Salle.

Flórez, R. (2005). Pedagogía del conocimiento. Bogotá, Colombia: McGraw Hill.

Flórez, R. (1999). Evaluación Pedagógica y Cognición. Bogotá, Colombia: McGraw Hill.

Flórez, R. y Tobón, A. (2001). Investigación educativa y pedagógica. Bogotá, Colombia: McGraw Hill.

Frei Betto. (1998). La obra del artista. Una visión holística del universo. Madrid, España: Ediciones Barbarroja.

Maturana, H. (1989). Emociones y lenguaje en educación y política. Santiago, Chile: Dolmen Ediciones.

Maturana, H. (1999). Transformación en la convivencia. Santiago, Chile: Dolmen Ediciones. 